\title{
¿Cuántos años monitorear el éxito de plantaciones con fines de restauración?: Análisis en relación al micrositio y procedencia de las semillas
}

\author{
How many years of monitoring are needed to determine plantation success in the context \\ of restoration?: Overview in relation to microsites and seed provenance
}

\author{
Nayara Simoes Macayo ${ }^{\text {a }}$, Daniel Renison ${ }^{\text {a* }}$

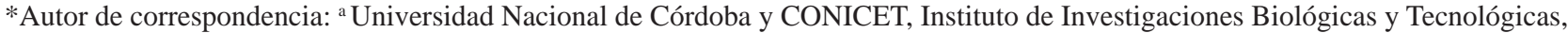 \\ Centro de Ecología y Recursos Naturales Renovables, Av. Vélez Sarsfield 1611, X5016GCA, Córdoba, Argentina, \\ drenison@conicet.gov.ar
}

\section{SUMMARY}

Field studies on the success of reforestation mostly report monitoring periods ranging from months to years, assuming that these periods are representative of the performance of individuals over longer time periods. We test this assumption by monitoring, for 15 years, a plantation of 585 trees of Polylepis australis produced from two seed provenances and planted in three types of microsites in the Mountains of Córdoba, Argentina. We monitored at year 0 (when planted), and years 1, 2, 3, 4, 5, 6, 10, 12 and 15 to evaluate whether the combined effects of the treatments on survival and height had similar patterns throughout time. We further determined if height in the different years of monitoring was a good indicator of the results of the last measurement year, the probability that the trees produce seeds at year 12, and the number, weight and viability of seeds produced. The difference in the height of the seedlings between the two provenances was significant since year 0 , while among microsites it became significant from year 3 onwards. Height was an unbiased indicator of all reproductive traits when measured from year 2 onwards. Finally, mortality was not affected by the treatments and reached a relatively constant rate from year 4 onwards. This study provides evidence that short-term evaluations (e.g., 2 years) may be representative of future behavior of the monitored individuals.

Key words: Polylepis australis, survival, growth, seed production, viability.

\section{RESUMEN}

Muchos estudios a campo sobre el éxito de reforestación reportan monitoreos que varían desde meses hasta unos pocos años, asumiendo que ese período es representativo del desempeño de los individuos a largo plazo. Aquí se propuso poner a prueba esta asunción, monitoreando durante 15 años una plantación de 585 árboles de Polylepis australis producidos con semillas de dos procedencias y plantados en tres tipos de micrositios en las Sierras Grandes de Córdoba, Argentina. Se monitoreó la supervivencia y la altura al año 0 (cuando se plantaron) y en los años 1, 2, 3, 4, 5, 6, 10, 12 y 15 para determinar si el patrón de resultados era similar en el tiempo, y se determinó si la altura en los distintos años de monitoreo era un indicador adecuado de la probabilidad de que los árboles produzcan semillas al año 12 y del número, peso y germinación de las semillas producidas. La diferencia en la altura de los plantines entre las dos procedencias fue significativa desde el año 0 , mientras que entre los micrositios se tornó significativa a partir del año 3 . La altura resultó ser un indicador no sesgado de todos los aspectos reproductivos medidos a partir al año 2. Finalmente, la mortalidad no estuvo afectada por los tratamientos y alcanzó una tasa relativamente constante a partir del año 4. Este estudio aporta evidencias de que las evaluaciones a corto plazo (por ejemplo, 2 años) pueden ser representativas del comportamiento futuro de los individuos monitoreados.

Palabras clave: Polylepis australis, supervivencia, crecimiento, producción de semillas, germinación.

\section{INTRODUCCIÓN}

Los sistemas montañosos de Sudamérica cuentan con una larga historia de uso antrópico lo cual ha inducido que grandes áreas de bosques se hayan perdido completamente o se encuentren degradadas por lo que la restauración de los bosques nativos y las actividades de investigación que la apoyan están en pleno crecimiento y han incorporano muchas de las técnicas desarrolladas por la industria forestal que necesitan adaptaciones a los nuevos contextos (Oliet y
Jacobs 2012). En particular, los monitoreos que se realizan para evaluar el éxito de plantaciones realizadas con el fin de restaurar bosques generalmente son de corto plazo y duran entre meses a un par de años (por ejemplo, 19 meses en Castro et al. 2002, seis meses en Pakkard et al. 2003) y no cuentan con un claro justificativo para la definición de la duración. Estos monitoreos son eficaces en el contexto de experimentos en plantaciones productivas donde generalmente se elimina la competencia de los pastos y hierbas y se homogenizan las condiciones de sitio usando grandes 
maquinarias. Esto es en parte debido a que estas intervenciones reducen el ruido ambiental y por ende realzan las diferencias entre situaciones experimentales, haciendo que las comparaciones a corto y largo plazo brinden patrones de resultados similares (por ejemplo: comparación entre 5 y 20 años en Sebben et al. 2005, comparación entre 0, 6 y 11 años en Bergin et al. 2008). Sin embargo, en el contexto de una restauración ecológica bajo muchas situaciones es deseable mantener la heterogeneidad ambiental y no es deseable el uso de grandes maquinarias y/o intervenciones en el ambiente, ya que se intenta recrear un ecosistema lo más similar posible al existente originalmente en el sitio, con la mayor parte de las especies de plantas originales (Lamb y Gilmour 2003).

En condiciones heterogéneas y bajo la influencia de la vegetación circundante es posible que los monitoreos a corto plazo no reflejen lo que ocurre a un plazo más largo. Por ejemplo, en los primeros estadios de crecimiento se puede registrar el fenómeno de facilitación con la vegetación circundante, pero a lo largo del tiempo esta vegetación puede actuar de competencia e inhibir el desarrollo de los individuos inicialmente favorecidos (Nuñez et al. 2009). De igual manera, sitios adecuados para la regeneración temprana pueden volverse inadecuados en estadios tardíos debido a la competencia por los recursos disponibles en el suelo (Casper y Jackson 1997). Debido a esto, se ha reclamado que en el contexto de proyectos de restauración los monitoreos deben ser a largo plazo especialmente en ambientes heterogéneos y cambiantes, ya que en estas situaciones y en algunas especies, los monitoreos a corto plazo pueden arrojar resultados poco confiables (Menges 2008).

La restauración de bosques nativos es cada vez más frecuente a nivel mundial (Lamb y Gilmour 2003) y en las Sierras Grandes de Córdoba, Argentina, existen al menos tres proyectos de reforestación con árboles nativos (Renison et al. 2013). Varios investigadores realizaron estudios tendientes a facilitar las técnicas de reforestación (Verzino et al. 2004, Renison et al. 2005, Soteras et al. 2014) y dos preguntas se desprenden de estas investigaciones: (i) ¿de qué procedencias deben ser las semillas para producir los plantines? y (ii) ¿̇en qué micrositios es mejor plantarlos? El objetivo de este trabajo es contribuir a determinar por cuántos años es necesario monitorear, a campo, los ensayos de reforestación en sitios heterogéneos, con el fin de obtener resultados fiables en relación al éxito a largo plazo. En una plantación experimental de Polylepis australis Bitter (Rosaceae) se evaluaron los efectos combinados de la procedencia de las semillas y de los micrositios de plantación durante los años 1, 2, 3, 4, 5, 6, 10, 12 y 15 para determinar: (i) si los patrones de supervivencia y crecimiento cambian a lo largo del tiempo de monitoreo y (ii) si existe una correlación entre el crecimiento vegetativo, la cantidad y calidad de las semillas producidas en el largo plazo. Se puso a prueba la hipótesis de que los monitoreos a corto plazo detectan ventajas iniciales entre los distintos tratamientos que luego se trasladan a los años posteriores. De ser cierta esta hipótesis, es de esperar que los análisis estadísticos realizados con datos de monitoreos a corto plazo (1 o 2 años) para la supervivencia y el crecimiento de los plantines arrojen resultados similares a los análisis, usando monitoreos a largo plazo. Además es de esperar que se registre una correlación positiva entre la tasa de crecimiento registrada a corto plazo y la cantidad y la calidad de las semillas producidas a largo plazo. Se considera importante analizar la producción de semillas debido a que los estadios iniciales en el ciclo de vida de las plantas, como la formación de frutos y semillas, la germinación de semillas y el posterior establecimiento y crecimiento de las plántulas, son fundamentales para la futura regeneración y todo monitoreo del éxito de una reforestación debe tener en cuenta la capacidad reproductiva de los árboles implantados.

\section{MÉTODOS}

Área de estudio y descripción de la especie. El paisaje de las Sierras Grandes de Córdoba, Argentina, consiste en mosaicos compuestos por distintos tipos de pastizales como los pajonales dominados por Poa stuckertii (Hack.) Parodi, Deyeuxia hieronymi (Hack.) Türpe y céspedes de Alchemilla pinnata Ruiz et Pav. y Carex fuscula d'Urv., bosques y matorrales de Polylepis australis, afloramientos rocosos naturales o con roca expuesta por erosión y pavimentos de erosión mayormente de origen antrópico (Cingolani et al. 2013). La precipitación media anual es de $854 \mathrm{~mm}$ y la mayor parte (83 \%) ocurre entre octubre y marzo. La temperatura media anual es de $8{ }^{\circ} \mathrm{C}$ y no se registran meses libres de heladas (Marcora et al. 2008).

Polylepis australis habita exclusivamente en las montañas del centro y noroeste argentino (Renison et al. 2013). Es un árbol o arbusto pequeño, de 3 a $14 \mathrm{~m}$ de altura, de corteza castaño anaranjada que se descascara en láminas muy finas (Renison et al. 2011). La polinización es anemófila y el fruto es uniseminado, seco y coriáceo. El fruto permanece dentro de un receptáculo castaño claro de 5 a $10 \mathrm{~mm}$ de largo y posee tres alas para su dispersión anemócora. Para este trabajo, se consideró como "semilla" a esta unidad dispersora.

Procedencia de los plantines, diseño experimental y monitoreo. Se recolectaron semillas de 40 árboles de una procedencia local denominada "Los Gigantes" $\left(31^{\circ} 25^{\prime} \mathrm{S}\right.$, $64^{\circ} 48^{\prime \prime}$ O; $2.000 \mathrm{~m}$ s.m.n.) y clasificada como un bosque degradado, y semillas de 40 árboles en el Parque Nacional Quebrada del Condorito (31 ${ }^{\circ} 40^{\prime}$ S, 64² $42^{\prime}$ O; $1.900 \mathrm{~m}$ s.n.m.) denominada "Condorito" y clasificada como un bosque conservado. Se realizó la siembra en marzo de 1998 y los plantines se mantuvieron en vivero hasta noviembre y diciembre del mismo año. La plantación se realizó en el sitio de la procedencia local 200 m s.n.m. más arriba que el promedio de los árboles semilleros seleccionados. Antes de realizar la plantación se alambraron 10 ha para excluir al ganado; el área había estado bajo pastoreo conti- 
nuo desde al menos 1960 y alrededor de $30 \%$ de los suelos se habían perdido por falta de cobertura vegetal adecuada (Renison et al. 2005, Cingolani et al. 2013).

Se seleccionaron los sitios de plantación para incluir la mayor variedad de ambientes respecto a la pendiente, orientación, substrato y vegetación circundante y se marcó cada sitio utilizando varillas metálicas numeradas. Se midió las características de micrositio más relevantes, como cobertura de distintos tipos de vegetación, profundidad y tipo de suelo, y mediante análisis de componentes principales, se clasificaron en tres tipos de micrositios (Renison et al. 2005): (1) roquedales y suelo sin cobertura vegetal, (2) céspedes degradados con muchas especies anuales y (3) céspedes en buen estado con pastos y hierbas bajas mantenidas así por el pastoreo que había en la zona hasta la construcción de la clausura. Se plantó el siguiente número de plantines en cada micrositio de las procedencias "Condorito" y "Los Gigantes": micrositio (1) 95 y 68; micrositio (2) 126 y 95; micrositio (3) 100 y 101; en total 585 ejemplares. Se regaron y se midieron la altura inicial de los plantines el día de la plantación (año 0).

Se monitoreó la supervivencia y el crecimiento de los ejemplares en los inviernos 1, 2, 3, 4, 5, 6, 10, 12 y 15 luego de ser plantados (de ahora en más años 1, 2, 3, etc.). Los monitoreos de 5 años fueron reportados en Renison et al. (2005) cuando los árboles en su mayoría aún no habían producido semillas. Además, al año 12 se registraron en una muestra de 362 árboles si habían producido semillas (sí o no) y se recolectaron manualmente todas las semillas producidas por cada ejemplar, se las guardó en bolsas de papel numeradas y se estimó su número, el peso por semilla y el porcentaje de germinación. Se estimó el número de semillas por conteo directo o mediante regla de tres simple pesando en balanza de precisión todas y 100 semillas de cada árbol (Pollice et al. 2013). Para cuantificar el peso por semilla se pesó una muestra de 50 semillas limpias de cada árbol ( $\mathrm{N}=127$ árboles). La germinación de las semillas se estimó para aquellos árboles que produjeron más de 50 semillas ( $\mathrm{N}=89$ árboles) en cápsulas de Petri con papel secante y algodón (50 semillas por cápsula y hasta tres cápsulas por árbol). Las cápsulas fueron mantenidas en una cámara con un fotoperíodo de 12 h de luz y 12 h de oscuridad, a una temperatura de $25^{\circ} \mathrm{C}$ y $15^{\circ} \mathrm{C}$ durante 60 días. Se regó y se registró la emergencia de radícula cada tres días.

Análisis de datos. Para las variables de respuesta mortalidad y altura de los plantines se realizaron los análisis para cada año de monitoreo. Para cada año de monitoreo (1 a 15) y para cada una de las dos variables de respuesta se armó un modelo lineal generalizado (GLM) con dos factores: (i) micrositio (tres niveles: sin vegetación, céspedes degradados y céspedes en buen estado), y (ii) procedencia (dos niveles: Los Gigantes y Condorito). Se modeló una variable de respuesta binomial (0-1) para la mortalidad y, en el caso de la altura, se incluyó únicamente los planti- nes con vida al año de monitoreo, por lo que los tamaños muestrales disminuyen con el tiempo.

Se determinó si la altura de los árboles en los sucesivos años del experimento era un indicador adecuado de su potencial reproductivo, realizando una regresión logística por año de monitoreo, donde la variable de respuesta fue la producción de semillas al año $12(0=$ no produjo; $1=$ produjo) y la variable explicatoria fue la altura en los respectivos años ( 0 a 12). Para todos los árboles que produjeron semillas en el año $12(\mathrm{~N}=127)$ se relacionó la altura con el número, peso y porcentaje de germinación de las semillas mediante correlación de Pearson. Se reportaron solamente las correlaciones para los 89 árboles de los cuáles se tenían todos los datos, incluido el porcentaje de germinación, ya que el patrón de resultados fue muy similar al obtenido en correlaciones que incluían todos los árboles posibles para cada variable.

Para cumplir con los supuestos de normalidad y homogeneidad de varianzas de los residuales, se aplicó la transformación de la raíz cuadrada para la altura del árbol, la transformación del logaritmo para el número de semillas y la transformación a arcoseno para el porcentaje de germinación de las semillas. Se realizaron las estadísticas utilizando el programa Infostat (2001).

\section{RESULTADOS}

Mortalidad y altura. La mortalidad fue máxima en el período del año 0 al 1 con un valor de 14 \% y disminuyó año a año hasta alcanzar una tasa relativamente constante, luego del año 4 de aproximadamente el $1 \%$ anual. No se registraron diferencias significativas en la mortalidad entre las dos procedencias ni entre los micrositios de plantación en ninguno de los años de monitoreo (todos los $P>0,05$; figura 1 , cuadro 1 ).

La altura de los plantines aumentó con el tiempo a una tasa promedio de 7,7 cm anuales. La diferencia en la altura de los plantines entre los tres tipos de micrositios se tornó significativa a partir del año 3 , y mantuvo siempre el mismo patrón de menor altura en los sitios originalmente sin vegetación, intermedia en los céspedes degradados, y de mayor altura en los céspedes bien conservados. En cambio, la diferencia en la altura de los plantines entre las dos procedencias fue significativa desde el año cero, es decir, que los plantines ya fueron plantados con diferencias en altura que se expresaron durante los nueve meses que estuvieron en invernadero. Las diferencias entre las dos procedencias aumentaron con los años de monitoreo hasta el año 15, y la procedencia local de Los Gigantes siempre presentó menor altura que la procedencia de Condorito (todos los $P<0,004$; figura 1, cuadro 1).

Producción de semillas. El 35 \% de los árboles produjo semillas en el año 12 ( $\mathrm{N}=362$ árboles). Se estimó una producción promedio de $770 \pm 99$ semillas por árbol, variando entre 2 y 16.332 semillas. El peso promedio por semilla 

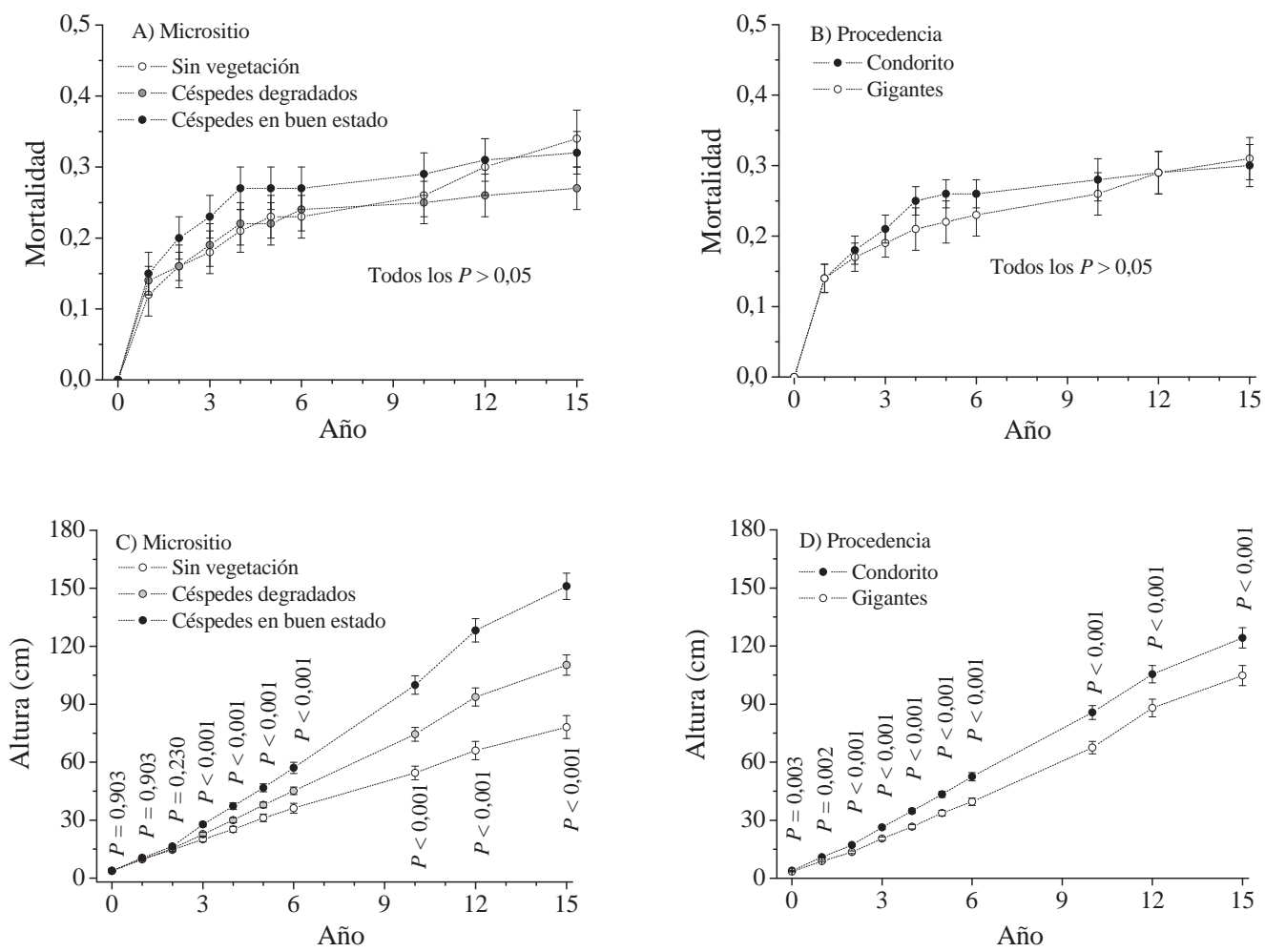

Figura 1. Mortalidades (A y B) y alturas (C y D) de Polylepis australis plantados a campo y monitoreados por 15 años en función del tipo del micrositio donde fueron plantados (A y C) y de la procedencia de las semillas (B y D). Los valores de $P$ son resultado de la prueba estadística para el año correspondiente.

Mortalities (A and B) and heights (C and D) of Polylepis australis samplings planted in a forest restoration site and monitored for 15 years as a function of the type of microsite where they were planted (A and $\mathrm{C}$ to the left) and as a function of seed provenance (B and $\mathrm{d}$ at the right). The $P$ values correspond to the statistical analyses of the corresponding year.

Cuadro 1. Análisis de mortalidad y altura de Polylepis australis para cada año de monitoreo. Para mortalidad se reporta el valor del Chi-cuadrado de Wald y el $P$ para un modelo lineal generalizado (MLG) con distribución binomial (supervivencias 0 o 1 , siempre en relación al año 0). En el caso de las alturas se aplicó la transformación de la raíz cuadrada y se reportan valores de $\mathrm{F}$ y $P$ para un ANCOVA. En ambos casos se consideraron dos factores (tipo de micrositio y procedencia).

Statistics for mortality and height of Polylepis australis saplings planted in a forest restoration site and monitored for 15 years, as determined for each of the monitored years separately. For mortality we report the Wald Chi-square and $P$ values of a Generalized Lineal Model (GLM) with assumption of binomial distribution (survivals of 0 or 1 - always in relation to year 0 ). For sapling heights we applied the square root transformation and report ANOVA F and $P$ values. In both cases we considered two factors (type of microsite and provenance).

\begin{tabular}{cccccccccc}
\hline & \multicolumn{4}{c}{ Mortalidades $(\mathrm{N}=585)$} \\
\cline { 2 - 10 } Año & \multicolumn{3}{c}{ Micrositio } & \multicolumn{2}{c}{ Procedencia } & \multicolumn{5}{c}{ Micrositio } & \multicolumn{2}{c}{ Procedencia } \\
\cline { 2 - 10 } & Valor de $\chi^{2}$ & $P$ & Valor de $\chi^{2}$ & $P$ & $\mathrm{~N}$ & Valor de $\chi^{2}$ & $P$ & Valor de $\chi^{2}$ & $P$ \\
\hline 0 & - & - & - & - & 585 & 0,102 & 0,90 & 8,634 & 0,003 \\
1 & 1,089 & 0,58 & 0,009 & 0,92 & 505 & 0,386 & 0,68 & 10,06 & 0,002 \\
2 & 1,382 & 0,50 & 0,283 & 0,59 & 483 & 1,473 & 0,23 & 14,44 & $<0,001$ \\
3 & 1,757 & 0,42 & 0,786 & 0,37 & 468 & 14,07 & $<0,001$ & 20,64 & $<0,001$ \\
4 & 2,390 & 0,30 & 1,577 & 0,21 & 448 & 19,37 & $<0,001$ & 28,66 & $<0,001$ \\
5 & 2,029 & 0,36 & 1,429 & 0,23 & 444 & 21,24 & $<0,001$ & 26,26 & $<0,001$ \\
6 & 1,423 & 0,49 & 0,733 & 0,39 & 441 & 23,85 & $<0,001$ & 28,85 & $<0,001$ \\
10 & 0,753 & 0,69 & 0,352 & 0,55 & 428 & 34,83 & $<0,001$ & 20,28 & $<0,001$ \\
12 & 1,432 & 0,49 & 0,000 & 0,99 & 415 & 37,47 & $<0,001$ & 13,47 & $<0,001$ \\
15 & 0,291 & 0,29 & 0,077 & 0,78 & 407 & 38,80 & $<0,001$ & 12,53 & $<0,001$ \\
\hline
\end{tabular}


fue de $0,0047 \pm 0,00018 \mathrm{~g}$ y varió entre 0,0004 y $0,010 \mathrm{~g}$ ( $\mathrm{N}=127$ árboles). El porcentaje promedio de germinación por árbol fue de 34,2 $\pm 1,9 \%$ y varió entre ejemplares con $0 \%$ y $88 \%$ de semillas germinadas ( $\mathrm{N}=89$ árboles).

La probabilidad de que los árboles produzcan semillas al año 12 no estuvo significativamente relacionada a la altura en el monitoreo del año 0, pero a partir del año 1 se registró una relación significativa y positiva que se mantuvo hasta el año 12 (regresión logística, todos los $P$ $<0,05$; cuadro 2). Las correlaciones entre la altura de los árboles con el número de semillas, su peso promedio y su porcentaje de germinación al año 12 presentaron un patrón inesperado, registrándose una correlación negativa en los primeros años que se volvió positiva y más marcada en los años posteriores (cuadro 3). Las correlaciones negativas al año 0 entre la altura de los árboles y las variables de semilla solo fueron significativas para la germinación de las semillas, y al año 1 no fueron significativas en ningún caso. A partir del año 2 las correlaciones fueron todas positivas, siendo significativas para el peso de las semillas, al año 3 se le agregó el número de semillas, y al año 6 la germinación de las semillas; patrón que se mantuvo hasta el año 12 con todas las correlaciones entre la altura de los árboles y las variables de semilla positivas y significativas.

Cuadro 2. Regresiones logísticas analizando como variable respuesta si los árboles produjeron o no semillas al año 12 ( $\mathrm{N}=362$ árboles) y como variable explicatoria la altura de los árboles al momento de la plantación (año 0) y a los años 1, 2, 3, 4, 5, 6, 10 y 12. Se reporta el valor del coeficiente, su error, el valor del Chi-cuadrado de Wald y el valor de $P$.

Statistics for the logistic regressions where the response variable was if the planted saplings produced seeds or not $(1$ or 0$)$ at year $12(\mathrm{~N}=362$ trees) and the explanatory variable was tree height at the day they were planted (year 0 ) and at years 1, 2, 3, 4, 5, 6, 10 and 12 . Note that tree height at year 1 was already a good indicator of its probability of producing seeds at year 12 . We report the value of the coefficient, its error, Walds Chi-squared and $P$ values.

\begin{tabular}{cccc}
\hline Año & Coeficiente de regresión & Chi cuadrado de Wald & Valor de $P$ \\
\hline 0 & 0,039 & 1,360 & 0,243 \\
1 & 0,057 & 11,338 & 0,001 \\
2 & 0,068 & 32,124 & $<0,001$ \\
3 & 0,065 & 46,171 & $<0,001$ \\
4 & 0,074 & 63,261 & $<0,001$ \\
5 & 0,071 & 72,253 & $<0,001$ \\
6 & 0,064 & 76,068 & $<0,001$ \\
10 & 0,042 & 88,411 & $<0,001$ \\
12 & 0,034 & 93,992 & $<0,001$ \\
\hline
\end{tabular}

Cuadro 3. Correlaciones entre los indicadores del éxito reproductivo medidos al año 12 y la altura de los plantines al momento de la plantación (año 0) y a los años 1, 2, 3, 4, 5, 6, 10 y $12(\mathrm{~N}=89$ árboles). Para cada correlación se muestra el coeficiente de Pearson y el valor de $P$.

Statistics for the correlations between the indicators of reproductive success of the planted trees at year 12 and their heights the day they were planted (year 0 ) and at years $1,2,3,4,5,6,10$ and $12(\mathrm{~N}=89$ trees). For each correlation we show Pearsons correlation coefficient and the $P$ value.

\begin{tabular}{ccccccc}
\hline Año & \multicolumn{2}{c}{ Número de semillas } & \multicolumn{2}{c}{ Peso por semilla } & \multicolumn{2}{c}{ Germinación de las semillas } \\
\hline & Coeficiente & Valor de $P$ & Coeficiente & Valor de $P$ & Coeficiente & Valor de $P$ \\
\hline 0 & $-0,078$ & 0,467 & $-0,048$ & 0,654 & $-0,213$ & 0,045 \\
1 & $-0,023$ & 0,828 & 0,170 & 0,111 & $-0,054$ & 0,613 \\
2 & 0,138 & 0,196 & 0,217 & 0,041 & 0,182 & 0,088 \\
3 & 0,259 & 0,014 & 0,299 & 0,004 & 0,208 & 0,055 \\
4 & 0,326 & 0,002 & 0,289 & 0,006 & 0,153 & 0,152 \\
5 & 0,342 & 0,001 & 0,284 & 0,007 & 0,189 & 0,077 \\
6 & 0,325 & 0,002 & 0,304 & 0,004 & 0,242 & 0,022 \\
10 & 0,474 & $<0,001$ & 0,304 & 0,004 & 0,215 & 0,043 \\
12 & 0,486 & $<0,001$ & 0,327 & 0,002 & 0,214 & 0,045 \\
\hline
\end{tabular}




\section{DISCUSIÓN}

La cantidad de años necesaria para detectar los efectos de los tratamientos en el éxito de reforestación con $P$. australis varió según el tratamiento a ser considerado y la variable de respuesta que se tuvo en cuenta. Consideraciones generales indican que la cantidad de años de monitoreo también depende de los tamaños muestrales y de la precisión de las mediciones (por ejemplo, peso seco en vez de altura los plantines). La variable mortalidad no hizo un aporte significativo a la pregunta de cuántos años monitorear una plantación, debido a que no fue afectada por ninguno de los tratamientos a lo largo de todo el experimento. Con un monitoreo mínimo de un año se hubiera llegado al mismo resultado estadístico que con monitoreos de 15 años, pero quedaría la duda sobre la necesidad de más tiempo para que sean visibles los efectos de los tratamientos. La supervivencia de P. australis fue tan alta como la encontrada en especies de árboles comerciales (Castro et al. 2002) y no fue afectada en gran medida por factores como adversidades climáticas, agentes bióticos, falta de riego o fertilización (Mesén y Vásquez 2009). Estos resultados indican una buena adaptación y potencial para la reforestación con esta especie nativa.

El monitoreo del crecimiento en altura indicó que en nuestro sistema y con el tamaño muestral usado, las diferencias significativas entre micrositios se detectan al año 3 y las diferencias entre procedencias ya se detectan en invernadero (año 0) y se mantienen a lo largo del tiempo. Otras plantaciones realizadas con fines de restauración en ambientes degradados también muestran que el desempeño inicial de las plántulas puede ser un buen indicador del comportamiento futuro (Souza y Batista 2004, Carpenter et al. 2004). El crecimiento de P. australis se vio favorecido en los micrositios más conservados y con mayor disponibilidad de recursos, por lo cual se recomienda intervención como obstrucciones al agua de lluvia o facilitación del crecimiento con leguminosas u otras plantas nodrizas (como en Castro et al. 2002, Carpenter et al. 2004) en los micrositios más degradados para que los árboles plantados normalicen sus tasas de crecimiento. Si bien en el área de estudio la exclusión del ganado y la reforestación produjeron incrementos en la cubierta vegetal y disminución de la compactación del suelo, aparentemente las mejoras aún no son suficientes para homogeneizar los crecimientos.

La ventaja inicial de los plantines de la procedencia Condorito se trasladó a campo durante los 15 años de monitoreo. En nuestro caso la procedencia no local de Condorito no presentó dificultades de adaptación, considerando que los 15 años incluyeron años con precipitaciones y temperaturas bastante variables, probablemente debido a que las condiciones climáticas, la altitud y latitud del sitio de recolección de las semillas son muy similares al área de plantación. En ensayos de procedencia con especies tropicales de rápido crecimiento (ejemplo: Vochysia guatemalensis, Mesén y Vásquez 2009) también se registró que se mantiene el patrón de crecimiento a corto plazo y a 18 años. De todas maneras, es importante considerar que en los ensayos de procedencia es necesario esperar mucho tiempo para estar seguro de los resultados (St. Clair y Johnson 2004) y quizá estos períodos de monitoreo aún no hayan sido suficientes para que sean notables las consecuencias de una falta de adaptación.

La altura de los árboles resultó un buen indicador de todos los aspectos reproductivos medidos recién al año 2 . En monitoreos más cortos se detectó una relación negativa entre altura y algunos aspectos reproductivos medidos años después, lo cual es curioso, difícil de explicar y merece más investigación, pero a partir del monitoreo del año 2 este patrón ya no se observó y se volvió positivo. El esfuerzo reproductivo de los ejemplares aumentó en relación al tamaño del árbol sugiriendo que los árboles que alcanzan las mayores alturas no lo hacen a expensas de una reducción de los recursos destinados a la reproducción y además los árboles más grandes poseen más reservas energéticas para destinar a los eventos reproductivos. Este patrón ya fue descripto para $P$. australis y otras especies vegetales (Pollice et al. 2013), pero en otros casos se observa que debido a diferencias en la forma de asignar recursos, los árboles que mejor crecen producen pocas semillas o de mala calidad (Sainz et al. 2007).

El porcentaje promedio de germinación de las semillas fue más alto que el registrado en Renison et al. (2004) y es importante realizar otros estudios para explicar estas diferencias así como estudios similares en más de una estación reproductiva para determinar las posibles interacciones con la variabilidad climática ya que las bajas tasas de germinación de P. australis dificultan la reforestación y afectan la regeneración natural de la especie. Por otro lado, el establecimiento y adaptación de los renovales también es fundamental para la futura regeneración; en este caso, los aspectos reproductivos medidos fueron en términos de cantidad y calidad de las semillas producidas (número, peso por semilla y germinación), considerando que estas variables son indicadoras de la probabilidad de reclutar potenciales plantines y que los mismos sobrevivan; las semillas de menor peso por ejemplo, además de que resultan menos viables, pueden afectar el crecimiento temprano de los plantines y pueden producir plántulas de menor aptitud y capacidad competitiva (Du y Huang 2008). De todos modos, sería importante estudiar a campo el establecimiento de la descendencia de los árboles de este experimento en particular, ya que también existe una estrecha asociación entre los factores del microhábitat así como el estado de conservación del sitio y el establecimiento y crecimiento de los plantines (Renison et al. 2005).

La recuperación de sitios degradados en las Sierras Grandes de Córdoba y en el área del experimento en particular son procesos lentos, por ello es necesaria la intervención y la reforestación es una medida importante de control de la erosión y restauración de la vegetación. Este estudio aporta evidencias de que las evaluaciones realiza- 
das a corto plazo (por ejemplo, 2 años) en una reforestación pueden ser indicativas del éxito a largo plazo. Además, los resultados de este trabajo refuerzan la conclusión de Renison et al. (2005) sobre la importancia de considerar la selección del origen de las semillas y de los micrositios de plantación a la reforestación para incrementar la tasa crecimiento y favorecer el éxito reproductivo e indican que para $P$. australis a edades tempranas se pueden hacer recomendaciones válidas sobre la selección de procedencias y micrositios.

\section{CONCLUSIONES}

Se aporta evidencias de que las evaluaciones a corto plazo (2 años) pueden ser representativas del comportamiento futuro de los individuos monitoreados de P. australis en estudios de procedencias y en estudios tendientes a determinar en qué micrositios es mejor plantar los plantines, incluso, bajo condiciones muy heterogéneas como las encontradas en contextos de restauración ecológica. Es necesario realizar evaluaciones formales en un mayor número de especies y con preguntas de investigación puntuales para otros ecosistemas forestales para determinar y consolidar la generalidad de esta afirmación.

\section{AGRADECIMIENTOS}

Al Club Andino Córdoba por brindar alojamiento en Los Gigantes. A CONICET y el ministerio de Ciencia y Técnica de la provincia de Córdoba por el financiamiento. A R Claverie, D Lasdica, J Domínguez, J Reis Simoes, D Eckstein, L Stoeckl, A Diván y A Ibáñez por la ayuda a campo. A todos ellos: muchas gracias.

\section{REFERENCIAS}

Bergin DO, MO Kimberley, CB Low. 2008. Provenance variation in Podocarpus totara (D. Don): Growth, tree form and wood density on a coastal site in the north of the natural range, New Zealand. Forest Ecology and Management 255: 1367-1378.

Carpenter FL, J Doland Nichols, RT Pratt, KC Young. 2004. Methods of facilitating reforestation of tropical degraded land with the native timber tree, Terminalia amazonia. Forest Ecology and Management 202: 281-291.

Casper B, RB Jackson. 1997. Plant competition underground. Annual Review of Ecology and Systematics 28: 545-570.

Castro J, R Zamora, JA Hodar, JM Gomez. 2002. Use of shrubs as nurse plants: a new technique for reforestation in Mediterranean mountains. Restoration Ecology 10: 297-305.

Cingolani AM, MV Vaieretti, MA Giorgis, N La Torre, JI Whitworth-Hulse, D Renison. 2013. Can livestock and fires convert the sub-tropical mountain rangelands of central Argentina into a rocky desert? The Rangeland Journal 35: 285-297.

Du Y, Z Huang. 2008. Effects of seed mass and emergence time on seedling performance in Castanopsis chinensis. Forest Ecology and Management 255: 2495-2501.
Lamb D, D Gilmour. 2003. Rehabilitation and restoration of degraded forests. Gland, Switzerland and Cambridge, UK. IUCN. 111 p.

Marcora P, I Hensen, D Renison, P Seltmann, K Wesche. 2008. The performance of Polylepis australis trees along their entire altitudinal range: implications of climate change for their conservation. Diversity and Distributions 14: 630-636.

Menges ES. 2008. Restoration demography and genetics of plants: when is a translocation successful? Australian Journal of Botany 56: 187-196.

Mésen F, W Vásquez. 2009. Genetic variation of provenances and progenies of Vochysia guatemalensis at 18 years of age in Sarapiquí, Heredia, Costa Rica. Agronomía Costarricense 33: 157-170.

Nuñez CI, E Raffaele, MA Nuñez, F Cuassolo. 2009. When do nurse plants stop nursing? Temporal changes in water stress levels in Austrocedrus chilensis growing within and outside shrubs. Journal of Vegetation Science 20: 1064-1071.

Oliet JA, DF Jacobs. 2012. Restoring forests: advances in techniques and theory. New Forests 43: 535-541.

Pakkad G, F Torrea, S Elliottb, D Blakesley. 2003. Selecting seed trees for a forest restoration program: a case study using Spondias axillaris Roxb. (Anacardiaceae). Forest Ecology and Management 182: 363-370.

Pollice J, P Marcora, D Renison. 2013. Seed production in Polylepis australis (Rosaceae) as influenced by tree size, livestock and interannual climate variations in the mountains of central Argentina. New Forests 44: 233-247.

Renison D, I Hensen, AM Cingolani. 2004. Anthropogenic soil degradation affects seed viability in Polylepis australis mountain forests of central Argentina. Forest Ecology and Management 196: 327-333.

Renison D, AM Cingolani, R Suarez, E Menoyo, C Coutsiers, A Sobral, I Hensen. 2005. The restoration of degraded mountain woodlands: Effects of seed provenance and microsite characteristics on Polylepis australis seedling survival and growth in Central Argentina. Restoration Ecology 13: 129-137.

Renison D, I Hensen, R Suarez. 2011. Landscape structural complexity of high-mountain Polylepis australis forests: A new aspect of restoration goals. Restoration Ecology 19: 390398.

Renison D, E Cuyckens, S Pacheco, GS Guzmán, HR Grau, P Marcora, G Robledo, AM Cingolani, J Dominguez, M Landi, L Bellis, I Hensen. 2013. Distribución y estado de conservación de las poblaciones de árboles y arbustos del género Polylepis (Rosaceae) en las montañas de Argentina. Ecología Austral 23: 27-36.

Sainz RC, JC Maldonado, MA Sáez, DS de Ron, MRC Santos, RA Miranda. 2007. Modelización del esfuerzo reproductor en ecología forestal: el ejemplo de Pinus halepensis mill. Cuadernos de la Sociedad Española de Ciencias Forestales 23: 141-146.

Sebben AM, AC Zanatto, ML Freitas, AS Sato, L Ettori. 2005. Genetic variation in Araucaria cunninghamii provenances in Luiz Antonio SP, Brazil. Crop Breeding and Applied Biotechnology 4: 1-8.

Seltmann P, I Leyer, D Renison, I Hensen. 2007. Variation of seed mass and its effects on germination in Polylepis australis: implications for seed collection. New Forest 33: 171-181.

Soteras F, D Renison, AG Becerra. 2014. Restoration of high altitude forests in an area affected by a wildfire: Polylepis 
australis Bitt. seedlings performance after soil inoculation. Trees 28: 173-182.

Souza FM, J LF Batista. 2004. Restoration of seasonal semideciduous forests in Brazil: influence of age and restoration design on forest structure. Forest Ecology and Management 191: 185-200.

St Clair B, R Johnson. 2004. Structure of genetic variation and implications for the management of seed and planting stock. In Riley LE, RK Dumroese, TD Landis (technical coordinators). National Proceedings: Forest and Conservation Nursery Associations-2003. USDA Forest Service Proceedings RMRS-P-33. p. 64-71.

Verzino G, J Joseau, M Díaz, M Dorado. 2004. Comportamiento inicial de especies nativas del Chaco Occidental en plantaciones en zonas de pastizales de altura de las Sierras de Córdoba, Argentina. Bosque 25(1): 53-67.

Recibido: 09.10.14

Aceptado: 07.05.15 